\title{
9. Suites et metamorphoses
}

P arce qu'elle démontre l'impact signifiant du projet encyclopédique au cœur des Lumières, mais aussi par son immense succès éditorial, l'Encyclopédie suscite une foule d'entreprises qui, dans la seconde moitié du XVIII ${ }^{e}$ siècle, la répètent, la prolongent ou la refondent. Ces entreprises sont intéressantes pour comprendre l'Encyclopédie elle-même : elles témoignent directement de la réception de l'œuvre en général et de chacun de ses articles (repris, annotés, amputés, complétés ou remplacés); elles permettent aussi de mesurer, par la façon dont le projet s'y transforme, une certaine latitude dans le sens qu'on pouvait alors donner à l'encyclopédisme ; elles révèlent enfin, au-delà des années 1780 , une mutation épistémologique qui fait basculer l'idée même d'encyclopédie dans l'âge nouveau des disciplines.

Parmi cette descendance foisonnante, il convient de distinguer plusieurs branches. Premièrement, les simples contrefaçons qui ne font qu'exploiter l'engouement pour l'œuvre mais qui en permettent une diffusion singulièrement élargie. Ensuite, les dictionnaires généraux, de langue ou universels, qui en exploitent certains apports : d'un côté, le Grand Vocabulaire Français publié entre 1767 et 1774 , qui utilise la nomenclature et les définitions de l'Encyclopédie; de l'autre, en 1771, la dernière édition du Dictionnaire universel de Trévoux, sur lequel les jésuites n'ont visiblement plus la main, qui emprunte largement, par un ironique retour des choses, à l'Encyclopédie, voire en importe carrément certains articles. Enfin et surtout, les projets qui constituent une reprise ou une continuation du programme encyclopédique lui-même. 
Dans ce troisième ensemble, viennent d'abord les nouvelles éditions toscanes "cum notis " : celle de Lucques, publiée entre 1758 et 1776, et celle de Livourne, qui paraît entre 1770 et 1775 . Le texte original y est reproduit mais avec des notes, plus occasionnelles que systématiques, tantôt érudites, tantôt italo-centriques, tantôt plus idéologiques. C'est aussi un Italien, mais installé en Suisse, qui s'engage à Yverdon dans une refonte complète de l'Encyclopédie, dont il rejoue toutes les étapes d'élaboration: F. B. De Felice élabore un système des connaissances profondément réorganisé (voir illustration), réunit une équipe internationale d'une quarantaine de collaborateurs, édite progressivement les volumes d'articles au format in-quarto (plus petit) pour lesquels le texte parisien sert de canevas, amplement retravaillé, puis

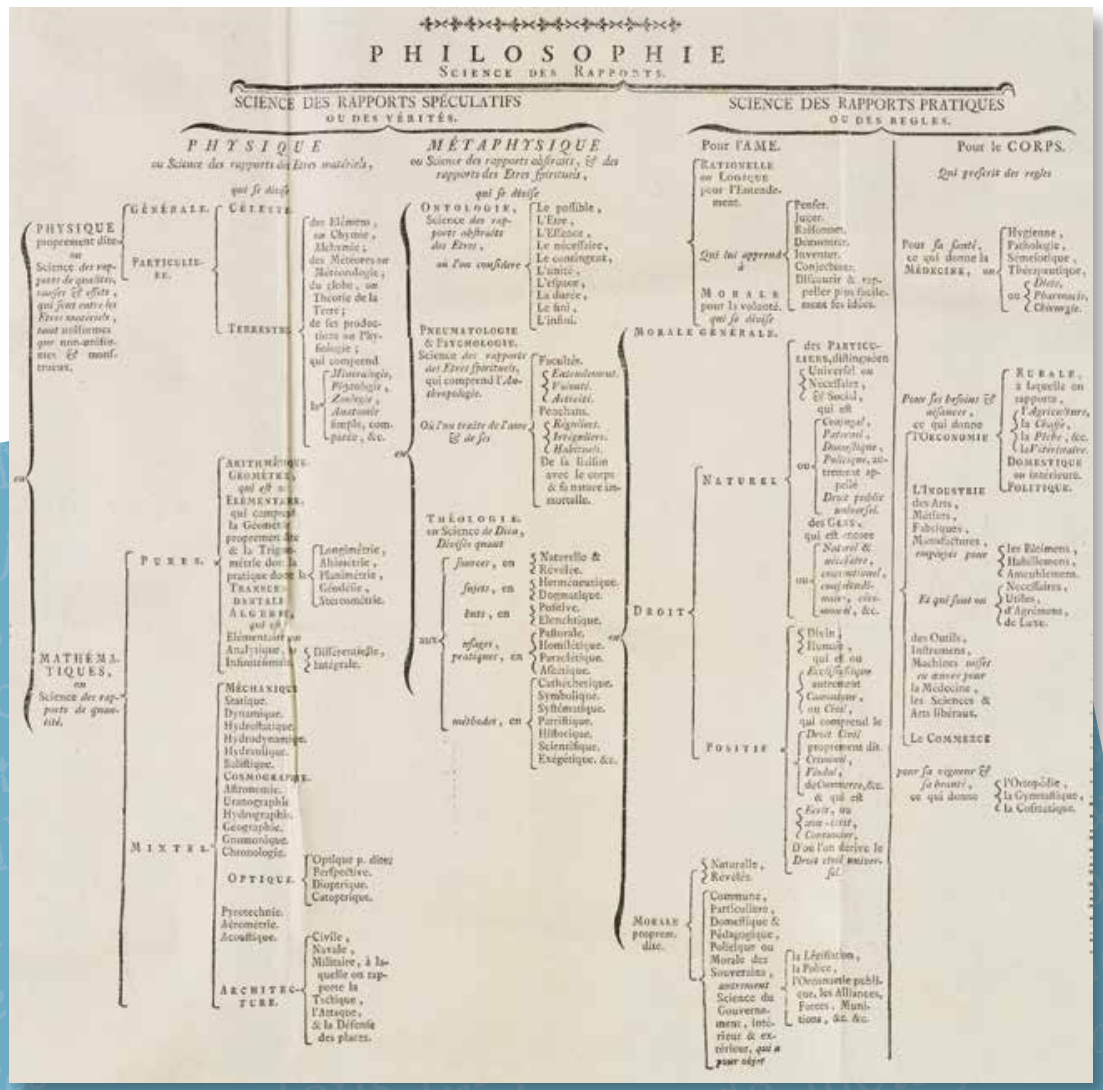

Au début du volume I de l'Encyclopédie d'Yverdon, le Système figuré des connaissances, entièrement repensé par De Felice et ses collaborateurs, témoigne d'une vision différente des liens entre les savoirs où, par exemple, la dimension religieuse s'inscrit au cour du dispositif (extrait : partie centrale ; BCU, Lausanne). 


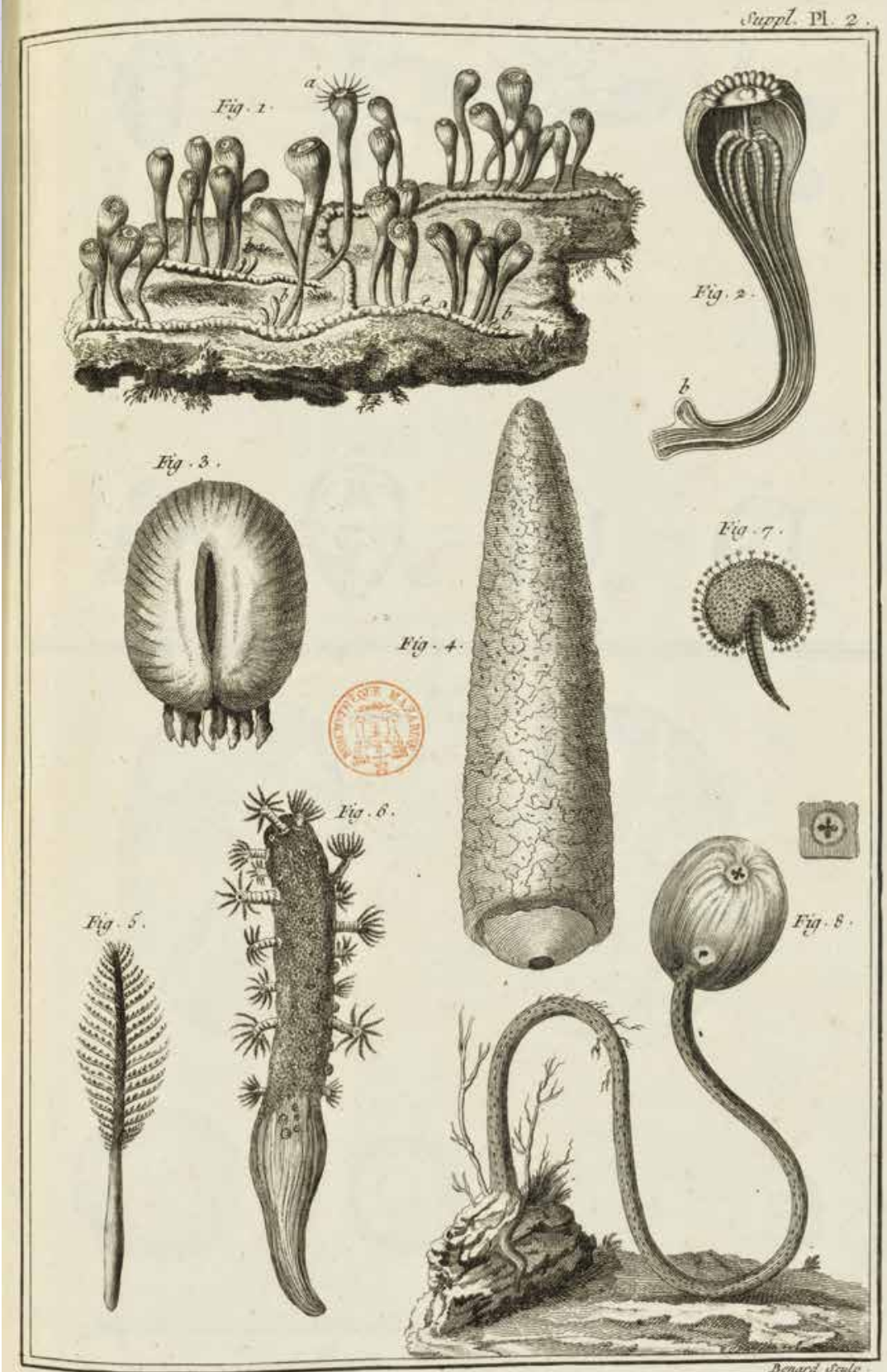

Histoire Naturelle, zoophites.

Planche dédiée aux zoophytes, publiée en 1777 dans le recueil de planches du Supplément à l'Encyclopédie. 
les volumes de planches, attentivement connectés aux articles. L'effort est placé sur la mise à jour des connaissances, sur une ouverture plus européenne des références, enfin sur une conception protestante des Lumières. Cette Encyclopédie dite "d'Yverdon " compte au total 58 volumes, parus entre 1770 et 1780, essentiellement diffusés en Suisse et dans l'Europe protestante. D'autres encyclopédies européennes ont par ailleurs subi diversement l'influence de celle de Diderot et D'Alembert, comme l'Encyclopæedia Britannica (deuxième et troisième édition) ou la Deutsche Encyclopädie (1807).

Une place à part doit être faite aux entreprises conduites par le libraire parisien Charles Joseph Panckoucke. Après avoir lancé le Grand Vocabulaire Français, déjà évoqué, il rachète, en 1768, les droits sur l'Encyclopédie ; il se lance d'une part dans des rééditions (in-folio de Genève et in-quarto de Genève et Neuchâtel) ; il suscite d'autre part l'idée d'un Supplément à l'Encyclopédie, qui sera dirigé par J. B. Robinet depuis Bouillon, avec une équipe également internationale de collaborateurs, et qui aboutit à la parution de quatre volumes d'articles et d'un volume de planches entre 1776 et 1777. Pour mieux articuler ces volumes à ceux de l'Encyclopédie, Panckoucke fait encore réaliser une Table analytique et raisonnée (1780), en deux volumes, par le pasteur bâlois Pierre Mouchon. Il convient de souligner que la présentation traditionnelle de l'Encyclopédie comme un ensemble incluant le Supplément et la Table provient de cette habile politique inclusive conçue par Panckoucke mais recouvre en fait des projets éditoriaux parfaitement distincts. Il faut noter aussi que les rééditions ou contrefaçons en formats in-quarto ou in-octavo (ce dernier format est encore plus petit) — qui démultiplient la diffusion de l'œuvre à la fin des années 1770 et au début de la décennie suivante - fondent en un seul dictionnaire les articles de l'équipe de Diderot et ceux du Supplément.

Panckoucke s'empare enfin, en 1780, d'un projet d'encyclopédie par ordre des matières qui, sous sa direction, va paraître à partir de 1782 sous le titre d'Encyclopédie méthodique. L'entreprise devient une sorte de monstre éditorial fractionné en une quarantaine de séries formant au total plus de 200 volumes dont le dernier paraîtra en... 1832 ! Les articles de l'Encyclopédie s'y trouvent éclatés (et, souvent, répétés dans plusieurs séries); chaque série travaille ce matériau de base à sa manière. L'encyclopédisme change de nature : il devient totalisation cumulative de connaissances organisées de façon indépendante, somme d'encyclopédies conçues d'abord par disciplines. Il s'éloigne ainsi irrémédiablement de l'ambition diderotienne d'une circulation encyclopédique à l'intérieur de l'ensemble des savoirs permettant d'appréhender les connaissances comme un tout, selon une logique universaliste caractéristique des Lumières. 